\title{
Criminología, prevención de la violencia y la agenda 2030 del desarrollo sostenible.
}

Recibido: 08-06-2021

Aceptado: 08-07-2021

\section{Resumen}

LaCriminologíaes unacienciamultidisciplinaria, que nos permite tener un universo de actuación en la sociedad, al ser un actor principal en la reducción de la violencia. Por lo cual, en el presente documento, abordaremos una breve revisión del contexto epistemológico de la Criminología, la estadística criminal en el estado de Nuevo León, para abordar la diferencia de la prevención de la violencia, del delito y/o delincuencia, su abordaje desde el marco legal así como sus principales enfoques teóricos, lo que nos lleva a conocer y analizar la Agenda

Cómo citar

Esquivel Hernández, A. M. CRIMINOLOGÍA, PREVENCIÓN DE LA VIOLENCIA Y LA AGENDA 2030 DEL DESARROLLO SOSTENIBLE. Revista Constructos Criminológicos. Recuperado a partir de https://constructoscriminologicos.uanl.mx/index.php/ cc/article/view/8

*https://orcid.org/0000-0003-1966-0175

Universidad de Autónoma de Nuevo León, México
2030 del Desarrollo Sostenible y las acciones que en este rubro realiza el gobierno mexicano, desde sus instrumentos diseñados para ello para finalmente reflexionar el papel que tiene la Criminología con sus conocimientos aportar a la construcción de las políticas públicas.

Palabras clave: Criminología, prevención, violencia, Agenda 2030.

\section{Abstract}

Criminology is a multidisciplinary science, which allows us to have a universe of action in society, being a main actor in reducing violence. In this document, we will address a brief review of the epistemological context of Criminology, criminal statistics in the state of Nuevo León, Mexico, to address the difference between prevention of violence, crime and/ or delinquency, its approach from the legacy framework as well as its main theoretical approaches. This will leads us to analyze the 2030 Agenda for Sustainable Development, and the actions that Mexican government carries 
out in this area, from its instruments designed for it to finally realize the role that Criminology has in the construction of public policies.

Keywords: Criminology, prevention, violence, 2030 Agenda.

Hoy en día la seguridad se vertió de diversos matices, al presentarse la pandemia del SARSCOV-2, se acentuaron nuevas criminalidades o formas de criminalización, como se ha presentado en diversos foros nacionales e internacionales, la coyuntura que la humanidad presenta, nos coloca en un repensar a donde nos dirigimos en la relación de la Criminología, la prevención de la violencia y la delincuencia así como las acciones que nuestro país despliega en relación con la Agenda 2030 del Desarrollo Sostenible vista desde el Objetivo: 16 que es el que nos ocupa en este documento.

\section{UNA VISIÓN DE LA CRIMINOLOGÍA Y CRIMINALIDAD}

En una sociedad cada vez más globalizada en la que los últimos dos años padece de un giro a todas sus estructuras a raíz de enfrentar una pandemia a la que no se encontraba preparada y ha desafiado a todas las políticas públicas antes vista a nivel internacional, a lo que nuestro país no ha quedado exento, por lo cual es necesario dar una breve revisión al mapa epistemológico de la Criminología, lo que desde sus bases nos permitirá entender la prevención de la violencia y/o el delito y su importancia para la Agenda 2030. En este contexto entendemos a la Criminología como:
Ciencia empírica e interdisciplinaria, que se ocupa del estudio del crimen, de la persona del infractor, la víctima y el control social del comportamiento delictivo, y trata de suministrar una información valida, contrastada, sobre la génesis, dinámica y variables principales del crimen contemplado éste como problema individual y como problema social-, así como sobre los programas de prevención eficaz del mismo, las técnicas de intervención positivas en el hombre delincuente y los diversos modelos o sistemas de respuesta al delito (GarcíaPablos 2006:33).

Por consiguiente, coincidimos con las bases epistemológicas de la Criminología expuestas por Palacios (2015: 440), donde desplegó un mapeo de la Criminología (Cuadro 1) en el cual se configuró de la siguiente manera:

a) Paradigmas: el del conflicto y el del consenso.

b) Ideologías: derecha, liberal e izquierda. c) Corrientes: Disuasión, Clínica, Interaccionista, Crítica e Integración Teorética esta última llamada la quinta corriente.

Por paradigma entendemos (Ardila, M., \& Marín, M., 2007, p. 36) a "un conjunto de valores y saberes compartidos colectivamente, es decir, usados, implícita o explícitamente, por una comunidad", por lo que al hablar del consenso, entendemos que los hombres deciden interactuar bajo ciertas reglas o moldes preestablecidos, por lo que quien deciden no seguir este modelo se pensaba que estaba bajo una influencia de orden biológico, como expone 
Cuadro 1. Mapa Epistemológico de la Criminología

\section{CRIMINOLOGÍA}

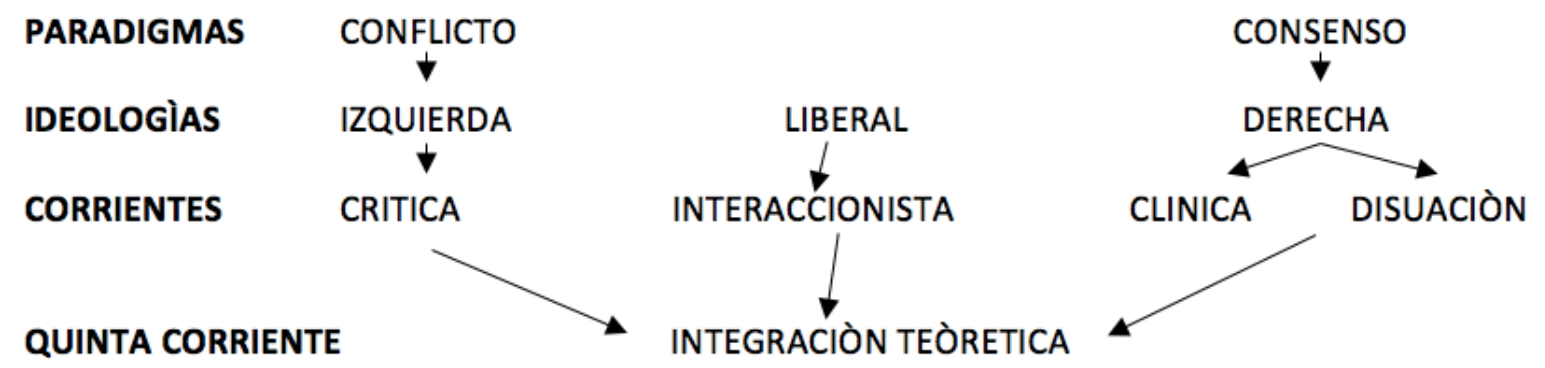

Fuente: Palacios P. (2015), Criminología Contemporánea, Introducción a sus fundamentos teóricos, INACIPE.

Lombroso en su obra: El Hombre Delincuente, donde como conocemos, la corriente de Derecha o Etológica expone al sujeto como el punto de explicación de la criminalidad, como factor causante de esta, al observarlo como un sujeto bio-psico-social, por lo que toma relevancia las Corrientes de tipo Clínico.

Así mismo la Disuasión expuesta bajo las teorías situacionales, que proponen diseñar espacios en los que se retraiga a los sujetos de cometer conductas antisociales, de la misma forma se entiende que al sujeto le influye el entorno, para realizar conductas antisociales y por consecuencia controlar la criminalidad.

Por otra parte, se encuentra el paradigma del Conflicto abordado por Marx al colocar a consideración las relaciones entre los obreros, los patrones y el Estado, lo cual propicia un cambio social, por lo que expone:

"La expropiación y el desahucio de una parte de la población rural, no solo deja a los obreros sus medios de vida y sus materiales de trabajo disponibles para que el capital industrial los utilice, sino que además crea el mercado interior". (Marx, 2009:120).
Bajo esta premisa la mayoría de la población se vuelve obrero subsistiendo con un salario asignado por el patrón, donde es la oferta y la demanda quienes suponen la base de la economía, dejando al Estado solo como espectador, ocasionando a nivel social que se plasme la desigualdad entre la riqueza generando clases sociales que pueden ostentar el poder.

De esta visión nace la corriente Crítica de la Criminología que en palabras de González y Sánchez (2010: 230) "se puede entender como el pensamiento que ubica la cuestión criminal como forma de una construcción social de las relaciones de dominación". Así, se deduce que las personas cometen conductas antisociales como consecuencia de que el Estado no brinda las condiciones necesarias para cubrir sus necesidades básicas entendidas, estas como alimentación, vestido, trabajo y educación; incluyendo que es el sistema penal quien designa lo que se considera delito, marcando bajo leyes y reglamentos lo que se considera conducta antisocial. En otros términos, es el Estado y su aplicación de la justicia penal quienes 
construyen la criminalidad configurando lo que hoy conocemos como violencia estructural a lo que indica La Parra y Tartosa (2003:57):

El término violencia estructural es aplicable en aquellas situaciones en las que se produce un daño en la satisfacción de las necesidades humanas básicas (supervivencia, bienestar, identidad o libertad) como resultado de los procesos de estratificación social, es decir, sin necesidad de formas de violencia directa.

Aquí, se despliega como concepto toral el control social, utiliza el control formal como forma de expresión, en el cual se fundamentan premisas para la actuación criminológica, por tal concepto se entiende:

Son los recursos y mecanismos de que disponen una determinada sociedad para asegurarse de la conformidad de los comportamientos de sus miembros a un conjunto de reglas y principios establecidos, así como las formas de reacción con las que se enfrentan las transgresiones. Se distinguen dos formas; control social formal, como el Derecho penal y control social informal (la familia, la escuela, la religión). (Vidaurri, 2018:45)

Continuando con esta descripción, la tercera ideología que se plantea, es la liberal esta nace a la luz de lo que conocemos como Estado Liberal, es la que da como origen a la corriente Interaccionista donde sus representantes más destacados son George H. Mead, Howard Becker, Erving Goffman, Edwin Lemert, Dennis Chapman.
Por último, la más reciente Corriente es la Integración Teorética, son el resultado de integrar diferentes elementos de las teorías precedentes, coherentes con el enfoque criminológico que considera que el delito es un evento multicausal (Herrero, Z.,2016:1). Esto permite, una explicación vista desde diversos ángulos, acrecentando la variedad de la información.

La amplitud del conocimiento criminológico, nos brinda la posibilidad de contribuir a disminuir la incidencia delictiva, a la que la Criminología está obligada a dar respuestas para su contención, aquí señalaremos que las estadísticas son variadas, dependiendo de la Institución que las presenta, así como la metodología utilizada, esta última también debe de ser un área de aportación por la Criminología.

Si tomamos en cuentalas estadísticas presentadas por la Secretaria de Seguridad y Protección Ciudadana según el Secretariado Ejecutivo del Sistema Nacional de Seguridad Pública de las carpetas de Investigación, con base a los delitos de impacto por cada 100,000 habitantes, en el periodo de diciembre 2018 a abril del 2021, para este trabajo colocamos las del 2020, las que existen hasta abril del 2021 y el ranking del Estado, que entre más cercano al número 1, más presente se encuentra el delito, con base a los años anteriormente mencionados, como vemos en la Tabla 1. 
Tabla 1. Carpetas de Investigación en el Estado de Nuevo León.

\begin{tabular}{|c|c|c|c|}
\hline \multicolumn{2}{|c|}{ CARPETAS DE INVESTIGACIÓN EN EL ESTADO DE NUEVO LEÓN } \\
\hline DELITO & 2020 & ABRIL 2021 & RANKING ABRIL 2021 \\
\hline Delitos de Impacto & 36,131 & 12,374 & 18 \\
\hline Homicidios dolosos & 847 & 310 & 16 \\
\hline Trata de personas & 48 & 44 & 5 \\
\hline Secuestro & 16 & 5 & 27 \\
\hline Robo de vehículos & 1685 & 790 & 19 \\
\hline Robo a casa habitación & 2527 & 805 & 8 \\
\hline Narcomenudeo & 3869 & 1553 & 7 \\
\hline Violencia familiar & 17940 & 6132 & 6 \\
\hline Feminicidios & 67 & 14 & 14 \\
\hline Violación & 779 & 268 & \\
\hline
\end{tabular}

Fuente: Secretariado Ejecutivo del Sistema Nacional de Seguridad Pública.

Como podemos observar los delitos que continúan al alza son la trata de personas, la cual se cuantifica por medio de las aprensiones, al igual que el narcomenudeo, el feminicidio, la violencia familiar, que son los que con la pandemia se han agravado por el confinamiento $y$ requieren de programas eficientes de prevención.

Otra estadística que se presenta por mes es la que nos divulga el Observatorio de Seguridad y Justicia (OSJ), el cual mide un total de 21 delitos por medio de las carpetas de investigación y al mes de abril del 2021, nos presenta lo siguiente: En esta otra medición encontramos los mismos delitos, lo que nos reafirma la presencia de la criminalidad, en la que es urgente que se trabaje de forma colaborativa y multidisciplinaria en su contención.

\section{LA PREVENCIÓN DESDE DIVERSOS MARCOS DE REFERENCIA}

Una de las grandes confusiones que aún hoy existen, es la mención de la prevención del delito y la prevención de la violencia y la delincuencia, lo que se traslada a discursos que impacta en la poca claridad de las acciones que se implementan y por consecuencia en la dificultad de la construcción del proyecto y la medición del mismo. Por lo cual exponemos diversas definiciones del concepto de la prevención del delito, según las Directrices de las Naciones Unidas para la Prevención del Delito (2010:2), "la prevención de delito engloba toda la labor realizada para reducir el riesgo de que se cometan delitos y sus efectos perjudiciales en las personas y la sociedad, incluido el temor a la delincuencia". 
Tabla 2. Carpetas de Investigación Primer Trimestre 2021, OSJ.

\begin{tabular}{|c|c|}
\hline \multicolumn{2}{|c|}{$\begin{array}{c}\text { PRIMER TRIMESTRE DE } 2021 \\
\text { ESTADO DE NUEVO LEÓN }\end{array}$} \\
\hline DELITOS A LA ALZA & DELITOS A LA BAJA \\
\hline Trata de personas $(\uparrow 229.69 \%)$ & Robo a negocio con violencia $(\downarrow 52.37 \%)$ \\
Robo de vehículos $(\uparrow 51.67 \%)$ & Feminicidio $(\downarrow 50.53 \%)$ \\
Narcomenudeo $(\uparrow 34.49 \%)$ & Robo a negocio $(\downarrow 46.99 \%)$ \\
Robo de vehículo con violencia $(\uparrow 27.43 \%)$ & Robo a transeúnte con violencia $(\downarrow 36.28 \%)$ \\
Lesiones dolosas $(\uparrow 4.89 \%)$ & Total de robos con violencia $(\downarrow 27.87 \%)$ \\
Delitos sexuales $(\uparrow 1.16 \%)$ & Total de robos $(\downarrow 27.26 \%)$ \\
Violencia familiar $(\uparrow 0.90 \%)$ & Robo a transeúntes $(\downarrow 27.15 \%)$ \\
& Robo a casa habitación $(\downarrow 25.57 \%)$ \\
& Secuestro $(\downarrow 18.60 \%)$ \\
& Extorsión $(\downarrow 10.62 \%)$ \\
& Violación $(\downarrow 10.42 \%)$ \\
& Homicidio doloso $(\downarrow 1.75 \%)$ \\
& Homicidio culposo $(\downarrow 1.26 \%)$ \\
\hline
\end{tabular}

Fuente: Observatorio de Seguridad y Justicia.

Por su parte Pérez \& Pérez (2006:120) la definieron como "el conjunto de políticas y de mecanismos orientados a evitar el nacimiento, desenlace, avance y reaparición de la criminalidad". Estas políticas, tendrán que ser incrustadas, dentro de la política general del Estado; siendo esta última definición con la que coincidimos ya que afirmamos que la prevención independientemente si es del delito o de la violencia, debe de ser desplegada en diferentes momentos de la criminalidad, ya que entendemos que la Criminología aborda no solo las conductas tipificadas como delito, sino todas las problemáticas que pueden contribuir a la criminalidad o al criminal.

Por lo tanto, pasamos a desplegar diversas categorías que nos permiten entender los diversos conceptos en la materia donde sobresalen la prevención especial y general como la más clásica, la prevención vista desde el marco jurídico y la prevención desde los enfoques teóricos de la misma.

\subsection{La Prevención Especial y General.}

La prevención especial y general se podría decir que son de las clásicas en el campo de la prevención estas emanadas de las teorías de la pena donde el Derecho Penal, la Filosofía, la Sociología, así como la Moral y la Ética han buscado la respuesta si el delito puede ser prevenido.

Para lo cual Hassemer \& Muñoz (2012:165) describen a las teorías preventivas de la pena, como "aquellas teorías que atribuyen a la pena la capacidad y la misión de evitar que en el futuro se cometan delitos". De aquí es donde surgen las variantes de prevención especial y general, se exponen de la siguiente forma:

1. Prevención especial: la que dirige su 
atención al delincuente concreto castigado con una pena, esperando que la pena tenga en él un efecto "resocializador", o por lo menos de "aseguramiento".

2. Prevención general: la que se dirige a la generalidad de los ciudadanos, esperando que la amenaza de una pena y, en su caso, la imposición o ejecución de la misma sirvan, por un lado, intimidar a los delincuentes potenciales (concepción estricta o negativa de la prevención general) y, por otro, para robustecer la conciencia jurídica de los ciudadanos y su confianza y fe en el Derecho.

Sin embargo, ambos tipos de prevención han comprobado su ineficacia como efectos preventivos con el paso de los tiempos como menciono Ruiz (2010:300) "hasta ahora hemos confiado exclusivamente la sanción, la represión y el castigo a la policía, por lo que, si queremos en verdad reducir el crimen, tenemos que acudir también a medios no coactivos".

\subsection{La Prevención en el Marco Jurídico}

Es importante hablar de la prevención del delito, hoy enmarcada en el término prevención de la violencia y la delincuencia, el cual definiremos más adelante, desde el marco normativo nacional, esto lo coloca en una validación jurídica. Por lo cual señalamos lo siguiente:

Primero observemos desde la Constitución Política de los Estados Unidos Mexicanos, que refiere en una parte del "Articulo 21...... el Sistema Nacional de Seguridad Pública, que estará sujeto a las siguientes bases mínimas: en el inciso c) La formulación de políticas públicas tendientes a prevenir la comisión de delitos".
Aquí entendemos que el Sistema Nacional se encuentra constituido por el Ministerio Público, las instituciones policiales de los tres niveles de gobierno y la guardia nacional, por lo que son los encargados de desplegar las acciones en la materia, razón por la cual las áreas encargadas de la prevención se encuentran por lo general dentro de estas instituciones, es importante mencionar que en la actualidad ya existen al menos en Nuevo León, Secretarias de Prevención de la Violencia, en municipios.

Otro elemento es la Ley General para la Prevención Social de la Violencia y la Delincuencia creada en el 2012 la cual define a la prevención social de la violencia y la delincuencia como:

Artículo 2.- La prevención social de la violencia y la delincuencia es el conjunto de políticas públicas, programas y acciones orientadas a reducir factores de riesgo que favorezcan la generación de violencia y delincuencia, así como a combatir las distintas causas y factores que la generan.

En esta ley encontraremos los tipos de prevención, los cuales son: Comunitaria, situacional, Psicosocial y Social, que coloca la referencia y bases para el desarrollo de las acciones en los programas desplegados en la materia.

Por consiguiente, pasamos a exponer los tipos de prevención referidos, por lo que en el artículo 8, la prevención comunitaria la expresa de la siguiente manera:

La prevención en el ámbito comunitario pretende atender los factores que generan 
violencia y delincuencia mediante la participación ciudadana y comunitaria y comprende:

I. La participación ciudadana y comunitaria en acciones tendentes a establecer las prioridades de la prevención, mediante diagnósticos participativos, el mejoramiento de las condiciones de seguridad de su entorno y el desarrollo de prácticas que fomenten una cultura de prevención, autoprotección, denuncia ciudadana y de utilización de los mecanismos alternativos de solución de controversias;

II. El mejoramiento del acceso de la comunidad a los servicios básicos;

III. Fomentar el desarrollo comunitario, la convivencia y la cohesión social entre las comunidades frente a problemas locales;

IV. La participación ciudadana y comunitaria, a través de mecanismos que garanticen su efectiva intervención ciudadana en el diseño e implementación de planes y programas, su evaluación y sostenibilidad, y

V. El fomento de las actividades de las organizaciones de la sociedad civil

La prevención comunitaria como lo mencionó Dammert (2005:133) "en la cual de acuerdo con sus objetivos se incluyen todas las iniciativas destinadas al barrio, entendido como destino de la política pública y ejecutor de las mismas". En este sentido se utilizaría el control social informal como una forma de control de la criminalidad.

Ahora bien, este argumento enfatiza la necesidad de la participación ciudadana en su papel más proactivo como parte de las políticas públicas que despliega el Estado para la prevención.
Por tal razón la prevención comunitaria nos ofrece ciertas ventajas al ejercer un control participativo y una validación por parte de la ciudadanía.

De igual forma en septiembre del 2015; México Evalúa público un estudio llamado Bases para la Construcción de un Índice de Cohesión Social Vecinal en el que concluyó lo siguiente:

Se observó que el mayor reto que enfrentan los vecinos de estos conjuntos habitacionales es desarrollar un comportamiento participativo. Otros retos importantes incluyen: generar confianza entre ellos, ayudarse mutuamente, identificarse con los otros miembros de la comunidad y desarrollar un sentido de pertenencia hacia el lugar en el que viven (México Evalúa:38).

Por lo expresado como menciono Pascual (2006:136) "la resolución de los problemas sociales implica la promoción de procesos de cambio a partir de metodologías participativas". La falta de consideración de la prevención comunitaria sería un retroceso en las intervenciones preventivas en este país, por lo cual es un reto que nos requiere innovación en estos procesos de la mano de los sustentos científicos.

Posteriormente pasamos a exponer la Prevención Situacional, esta es una de las más aplicadas en México y cuenta con sustento teórico y metodologías para su aplicación. Por tal motivo Medina (2010:24) menciono como "el espacio defendible se ha convertido en una herramienta más de la prevención situacional la policía orientada a la solución de problemas". 
Es decir, convierte al delito en una opción utilitarista, selectiva y racional en el que las causas no tienen mayor importancia sino es la actuación que se tiene que tener sobre ellos al disminuir la oportunidad que se puedan presentar. De lo anteriores se desprenden teoría que se aplican en este tipo de prevención las cuales son las siguientes:

Primero la Teoría de la Opción Racional: aquí la base de la desviación es el cálculo de maximizar el placer y minimizar el dolor; el control social como un castigo racional, la disuasión como objetivo de control social, el control de los actos y no de los actores.

Segundo la Teoría de las Ventanas Rotas: aquí el comportamiento sin reglas y sin vigilancia es señal evidente para los ciudadanos de que el área no es segura, el temor domina a quien habita estas zonas o a las que, por alguna razón circulan en sus calles. (Orellana, 2012:123).

Tercero la Teoría de las Actividades Rutinarias: afirma que el delito predatorio ocurre cuando un delincuente potencial y un objeto propicio coinciden en tiempo y lugar, sin la presencia de un guardián capaz de imponer el orden. (Clarke \& Eck, 2008:76)

Estas son algunas de las teorías que sustentan este tipo de prevención, pero en la Ley anteriormente mencionada encontramos en lo referente a la prevención situacional, lo siguiente:

Artículo 9. La prevención en el ámbito situacional consiste en modificar el entorno para propiciar la convivencia y la cohesión social, así como disminuir los factores de riesgo que facilitan fenómenos de violencia y de incidencia delictiva.

A manera de corolario, se dirá que este tipo de prevención tiene un impacto fuerte en las políticas que se ejercen en prevención del delito y/o de las violencias, ya que por su inmediatez suelen ser usadas ampliamente en los programas de gobierno, esta tiene un impacto inmediato en el conocimiento de la comunidad, al ser visible, es altamente propuesto por las autoridades.

Otro tipo propuesto en la Ley es la prevención psicosocial que como lo expone el término va enfocada en el sujeto desde su desarrollo individual que impacta en su interactuar en la sociedad, por lo que la Ley General la expone en su artículo 10 como:

La prevención en el ámbito psicosocial tiene como objetivo incidir en las motivaciones individuales hacia la violencia o las condiciones criminógenas con referencia a los individuos, la familia, la escuela y la comunidad, que incluye como mínimo lo siguiente:

I. Impulsar el diseño y aplicación de programas formativos en habilidades para la vida, dirigidos principalmente a la población en situación de riesgo y vulnerabilidad;

II. La inclusión de la prevención de la violencia, la delincuencia y de las adicciones, en las políticas públicas en materia de educación, y III. El fortalecimiento de las capacidades institucionales que asegure la sostenibilidad de los programas preventivos".

Este tipo de prevención incide directamente sobre los factores endógenos y exógenos, 
propuestos desde la Criminología Clínica, los cuales pueden incidir en la tendencia a cometer conductas antisociales; por lo que en general podríamos decir que se debe de considerar este tipo de prevención ya que colabora en la construcción de tejidos sociales más sanos, al atender al sujeto de forma individual o colectiva según será la línea de actuación.

Finalmente, en el orden jurídico tenemos a la prevención social esta es un esquema progresivo donde su enfoque es visto de una forma multidisciplinaria y transversal al intervenir en modificar las condiciones sociales de la comunidad al generar proyectos de vida.

Desde la Ley mencionada encontramos en su artículo 7 lo siguiente:

La prevención social de la violencia y la delincuencia en el ámbito social se llevará a cabo mediante:

I. Programas integrales de desarrollo social, cultural y económico que no produzcan estigmatización, incluidos los de salud, educación, vivienda, empleo, deporte y desarrollo urbano;

II. La promoción de actividades que eliminen la marginación y la exclusión;

III. El fomento de la solución pacífica de conflictos;

IV. Estrategias de educación y sensibilización de la población para promover la cultura de legalidad y tolerancia respetando al mismo tiempo las diversas identidades culturales. Incluye tanto programas generales como aquéllos enfocados a grupos sociales y comunidades en altas condiciones de vulnerabilidad, y

V. Se establecerán programas que modifiquen las condiciones sociales de la comunidad y generen oportunidades de desarrollo especialmente para los grupos en situación de riesgo, vulnerabilidad, o afectación.

Esta busca fortalecer los programas educativos, deportivos, culturales y de salud, así como el acceso a programas de empleo y de emprendimiento que contribuyan a generar más y mejores ingresos, la cual se enfocan como prioridad en los niños y jóvenes de colonias marginadas o comunidades con rangos de pobreza, fortalece el sentido de pertenencia y la participación ciudadana, lo cual junto con otros tipos de prevención abonara en la cultura de paz y la legalidad.

Por lo tanto, sostenemos que, desde la normativa jurídica nacional se sientan las bases para sustentar el actuar en la materia, lo que permite un lineamiento y homologación de las líneas de actuación, independientemente de los cambios de administraciones o estructuras desde la administración pública, así como ampliar la capacidad de gestión de diversas organizaciones.

\subsection{Prevención de la Violencia: Enfoques Teóricos}

En los últimos años se ha implementado la prevención de la violencia desde diversos enfoques, principalmente el de tipo epidemiológico y el ecológico, por lo que daremos un breve repaso de los mismos.

Primeramente, hablaremos del enfoque epidemiológico, el cual surge de la atención a la salud por lo que la Organización Mundial de la Salud la define como "el estudio de la 
distribución y los determinantes de estados o eventos (en particular de enfermedades) relacionados con la salud y la aplicación de esos estudios al control de enfermedades y otros problemas de salud". Motivo por el cual, en 1993, dada la magnitud y complejidad de la violencia, la Organización Panamericana de la Salud, expuso lo siguiente:

Las conductas violentas en la Región de las Américas constituyen un grave problema de salud pública y son causa de deterioro de la calidad de la vida.

Sus consecuencias para la salud y el bienestar de las colectividades, su equilibrio social y su estabilidad económica reviste proporciones alarmantes.

De ahí que Foro de Expertos en Seguridad Ciudadana de Chile (2004) y el Banco Mundial, implementan los niveles de intervención a llevar a cabo en los planes, programas y proyectos en la materia, donde se establecen los siguientes según la Subsecretaría de Prevención y Participación Ciudadana (SEGOB) y del Instituto Nacional Demócrata para Asuntos Internacionales (NDI), en el 2018.

1. Primaria: Dirigido a grupos de la población en general, con el fin de reducir los factores de riesgo y aumentar los que protegen a las personas de ser víctimas o victimarios.

2. Secundaria: Está destinada a evitar el desarrollo de conductas de riesgo de la violencia en grupos con un alto riesgo de presentar este tipo de comportamientos. Son intervenciones focalizadas que tienen como destino a poblaciones en situación de vulnerabilidad, tales como niñez, adolescencia, mujeres, personas en situación de discapacidad, población migrante, etc.

3. Terciaria: Son las intervenciones centradas en la atención a largo plazo con posterioridad a los actos violentos. Estas intervenciones pueden estar dirigidas tanto a la víctima como al victimario, con el principal fin de evitar la reincidencia y dar tratamiento al acto violento cometido (como la rehabilitación y reintegración, e intentos por reducir los traumas o la discapacidad de larga duración asociada con la violencia).

Colocar los niveles de intervención en el diseño de los programas o proyectos, nos permite tener claro las acciones a llevar a cabo, lo cual nos Ileva a formular de forma más clara los objetivos e indicadores, del mismo.

En segundo lugar, hablamos del enfoque ecológico, este enfoque toma fuerza tras la definición por parte de la OMS (2002), en el Informe Mundial sobre la Violencia y la Salud que entiende a "la violencia como la interacción de las personas en diferentes niveles y contextos, mediante el análisis de sus características individuales, relaciones cercanas, vínculos comunitarios e inserción social".

Esta nos Ileva a destacar las causas de la violencia y coloca en el contexto el conocimiento de los factores de riesgo y de protección, al definir los factores de riesgo como características que aumentan la probabilidad de que una persona se involucre en el crimen y la violencia (ya sea como víctima o agresor). De igual forma la Agencia de los Estados Unidos para el Desarrollo Internacional (USAID), define los 
Tabla 3. Enfoques de Prevención de la Violencia

\begin{tabular}{|c|c|c|}
\hline \multicolumn{2}{|c|}{ ENFOQUES EN PREVENCIÓN } \\
\hline \multirow{2}{*}{ EPIDEMIOLÓGICO } & Niveles de Intervención & $\begin{array}{c}\text { Primario } \\
\text { Secundario } \\
\text { Terciario }\end{array}$ \\
\hline \multirow{2}{*}{ ECOLÓGICO } & Factores de Riesgo & Individual \\
& Factores de Protección & Familiar/relacional \\
& & Comunitario \\
& & Sociales \\
\hline
\end{tabular}

Fuente: Elaboración propia.

factores de protección como "los activos o las características que disminuyen la probabilidad de que ocurran la violencia y la delincuencia" (USAID, 2015:43).

Estos dos factores presentan niveles de aparición Ios cuales son: Individual, Familiar/relacional, Comunitario y Sociales, partiendo que estos interactúan entre sí, o pueden estar presentes más unos que otros, en lo particular cuando realizamos un diagnóstico identificar los de protección conlleva un análisis profundo de los entornos, donde cada entorno presenta sus particularidades.

Ambos enfoques (Tabla 1) requieren de un nivel de conocimientos y preparación para su implementación y diseño, en los años que se ha trabajado en el tema entendemos que la prevención de la violencia necesita de conocimiento teórico y práctico para llevar a implementaciones cada vez más exitosas, que bajo una evaluación rigurosa se puedan considerar como buenas prácticas.
Por último, nos encontramos convencidos que la prevención en nuestra materia debe de ser un binomio entre la prevención del delito, visto desde las conductas tipificadas como tales y la prevención de la violencia, donde se observan las conductas que no siempre caen en este rubro, enfocándose a las causas de la misma. Lo que si puntualizamos es la importancia que bajo un diagnóstico se diseñe el programa en el que se desglosen los proyectos adecuados a cada contexto, esto permitirá según estos, colocar las acciones necesarias y dar respuestas a las necesidades de cada comunidad.

\section{EL MARCO INTERNACIONAL: LA AGENDA 2030 DEL DESARROLLO SOSTENIBLE}

Al hablar de la prevención de la violencia y el delito, desde el punto de vista internacional, retomemos algunos antecedentes, en septiembre del 2000, en la ciudad de Nueva York, se celebró la Cumbre del Milenio de las Naciones Unidas que da como resultado ocho Objetivos de Desarrollo del Milenio, que tenían 
como vigencia hasta el año 2015. Al término de estos las Naciones Unidas México, mencionan:

Se colaboró con el Gobierno en rubros que impactan el logro de los ODM en materia de: combate a la pobreza, seguridad alimentaria, perspectiva de género en planes y presupuestos, promoción del ejercicio de los derechos de la infancia, incremento en el acceso a servicios de salud materna y prevención del VIH/Sida, así como el fomento de una economía verde y el acceso a servicios básicos en áreas urbanas. De los 51 indicadores en los que México comprometió esfuerzos, se reportó cumplimiento total en 37 de ellos.

Lo anterior merece una revisión de los datos anteriormente mencionados, los cuales se encuentran en el portan de Naciones Unidas México, para conocer puntualmente su evaluación a los ocho objetivos, ante esto es claro que nuestro país tiende a desarrollar políticas que buscan cumplir con los estándares internacionales a los que está suscrito, señalamos que los retos subsecuentes son altos. Ante esto las Naciones Unidas al Ilegar el 2015, configuran lo que hoy conocemos como Los Objetivos de Desarrollo Sostenible (ODS) que son un conjunto de 17 objetivos y 169 metas destinadas a resolver los problemas sociales, económicos y ambientales que aquejan al mundo, cubriendo a partir de ese momento, los próximos 15 años (2015-2030).

Los ODS sustituyen a los Objetivos de Desarrollo del Milenio (ODM) que ya expiraron, colocando nuestra atención por el tema que nos ocupa en el siguiente:
Objetivo 16. Promover sociedades pacíficas e inclusivas para el desarrollo sostenible, facilitar el acceso a la justicia para todos y construir a todos los niveles instituciones eficaces e inclusivas que rindan cuentas

El objetivo mencionado contiene objetivos específicos, colocando sus espacios de aplicación los cuales se expresan a continuación:

16.1 Reducir significativamente todas las formas de violencia y las correspondientes tasas de mortalidad en todo el mundo 16.2 Poner fin al maltrato, la explotación, la trata y todas las formas de violencia y tortura contra los niños

16.3 Promover el estado de derecho en los planos nacional e internacional y garantizar la igualdad de acceso a la justicia para todos 16.4Deaquía 2030, reducirsignificativamente las corrientes financieras y de armas ilícitas, fortalecer la recuperación y devolución de los activos robados y luchar contra todas las formas de delincuencia organizada

16.5 Reducir considerablemente la corrupción y el soborno en todas sus formas 16.6 Crear a todos los niveles instituciones eficaces y transparentes que rindan cuentas 16.7 Garantizar la adopción en todos los niveles de decisiones inclusivas, participativas y representativas que respondan a las necesidades

16.8 Ampliar y fortalecer la participación de los países en desarrollo en las instituciones de gobernanza mundial

16.9 De aquí a 2030, proporcionar acceso a una identidad jurídica para todos, en particular mediante el registro de nacimientos 16.10 Garantizar el acceso público a 
la información y proteger las libertades fundamentales, de conformidad con las leyes nacionales y los acuerdos internacionales

16. a Fortalecer las instituciones nacionales pertinentes, incluso mediante la cooperación internacional, para crear a todos los niveles, particularmente en los países en desarrollo, la capacidad de prevenir la violencia y combatir el terrorismo y la delincuencia

16. b Promover y aplicar leyes y políticas no discriminatorias en favor del desarrollo sostenible

En este sentido nuestro país asumió el compromiso de implementar la agenda, por lo cual en la Estrategia Nacional para la Implementación de la Agenda 2030 en México, PARA NO DEJAR A NADIE ATRÁS: Por el bien de todos, primero los pobres, el cuidado del medio ambiente y una economía incluyente, publicada en noviembre del 2019, menciona en lo referente al Objetivo 16. Promover Sociedades Justas, Pacíficas e Inclusivas, lo siguiente:

En 2030, se habrá consolidado en México una cultura de servicio público fundamentada en la protección, garantía y promoción de los derechos humanos, la transparencia, la rendición de cuentas y la participación ciudadana, orientada al cumplimiento de las demandas públicas. Las instituciones del país -en todos los niveles- cumplirán con estándares de eficacia y transparencia, y rendirán cuentas sistemáticamente a la ciudadanía. Se habrá restaurado la confianza plena entre el gobierno y la sociedad, basada en la eficacia gubernamental y la participación de la ciudadanía en la deliberación y la toma de decisiones sobre los asuntos públicos, así como en la certidumbre que brinden las instituciones públicas a partir de un actuar enmarcado en la legalidad, la integridad y una sólida ética pública (Presidencia de la Republica, 2019a.).

En la estrategia anteriormente mencionada establece como prioridades o cambios necesarios para el cumplimiento de la Agenda, los siguientes:

- Erradicar la corrupción, el dispendio y la frivolidad.

- Recuperar el Estado de derecho

- Separar el poder político del poder económico

- Cambiar el paradigma en seguridad

- Transitar hacia una democracia participativa pública (Presidencia de la Republica, 2019b.).

Dentro de estos cambios necesarios ampliamos el de cambiar el paradigma de seguridad, en el que se menciona lo siguiente:

Cambiarlas medidas deguerrapor unapolítica de paz y seguridad integral que ataque la raíz misma del descontrol delictivo y la pérdida de seguridad, y que tenga como objetivo inmediato la reducción del índice delictivo. Esto implica erradicar la corrupción en la procuración de justicia, así como garantizar empleo, educación, salud y bienestar para todas las personas. Se tendrán que respetar plenamente los derechos humanos, regenerar la ética de las instituciones y de la sociedad, reformular el combate a las drogas, 
emprender la construcción de la paz, y recuperar y dignificar las cárceles. Asimismo, es necesario articular la seguridad nacional, la seguridad pública y la paz; establecer la Guardia Nacional; e instituir coordinaciones nacionales, estatales y regionales que sean efectivas pública (Presidencia de la Republica, 2019c.).

Este punto resulta interesante para el análisis del contexto, dejando en un rango amplio para su aplicación, dentro del contexto de la obligatoriedad que tiene nuestro país, por lo que revisemos el Programa Sectorial de Seguridad y Protección Ciudadana 2020-2024, publicado el 2 de julio del 2020 en el Periódico Oficial de la Federación, que nos muestra las directrices para llevar a cabo en materia de prevención de la violencia y el delito, en este se encuentran los cinco objetivos prioritarios:

- Objetivo prioritario 1: Mejorar las condiciones de seguridad en las regiones del territorio nacional para construir la paz.

- Objetivo prioritario 2: Contribuir al fortalecimiento del diseño e implementación de políticas públicas en materia de prevención de la violencia y el delito en el territorio nacional.

- Objetivo prioritario 3: Impulsar la reinserción social de las personas privadas de la libertad en centros penitenciarios con enfoque de respeto a los derechos humanos, inclusión y perspectiva de género, diferenciada e intercultural.

- Objetivo prioritario 4: Fortalecer las capacidades tecnológicas que permitan a las instituciones de seguridad de los tres órdenes de gobierno el intercambio seguro de la información en la generación de inteligencia, prevención y persecución del delito.

- Objetivo prioritario 5: Fortalecer la Gestión Integral de Riesgos para construir un país sostenible, seguro y resiliente. (DOF. 2019)

Este programa, nos muestra fallas muy visibles, primeramente, encontramos que no se realizó un diagnóstico como tal para su construcción, no vemos una estructura clara, lo cual en los últimos años quienes trabajamos en el tema, hemos insistido en que sin metodología no hay prevención, de la misma forma, las diversas acciones tanto las policiales como las comunitarias, no parecen llevar una conexión clara, se habla por ejemplo que los homicidios "habrán disminuido en un 50\%", un reto poco probable de cumplir cuando esto lleva ya varios sexenios en aumento, sabemos que los indicadores deben de ser realistas y viables en su construcción.

Ante este documento ya reaccionaron algunas organizaciones, por ejemplo, Causa Común (2020:9) menciona al respecto "Gobierno Federal no puede explicar de manera rigurosa, coherente y precisa, en qué consisten sus definiciones para enfrentar la situación de inseguridad, violencia y crimen más grave de los últimos cien años".

En este sentido entendemos que la configuración de políticas públicas en materia de seguridad debe de surgir de un diagnóstico bajo una metodología riguroso, con un sustento científico como ya lo marcan las construcción de la 
política criminal y la política pública, lo que nos muestran los documentos anteriormente citados, es una clara visión panorámica de lo local a lo internacional, lo que nos lleva a una grave desarticulación en las acciones que los gobiernos deben de emplear, ya que si la política criminal no es clara, la política pública tiende a fallar, donde urge la visión criminológica.

\section{CONCLUSIONES}

Las condiciones de la sociedad a raíz de la pandemia, han cambiado, hoy se encuentra con factores de riesgo más grandes, las condiciones económicas que generan las brechas entre clases se hacen cada vez más evidentes, tras las tasas de desempleo en el país, la situación escolar, se vio afectada, tras estar sujetos a dispositivos electrónicos para continuar con la instrucción escolar, las condiciones de salud mental se agravaron por diversas razones como la sana distancia, el confinamiento, la desesperanza y el duelo de muchas familias.

Todo lo anterior trajo como consecuencia que violencias que ya se presentaban se enfatizaron más, como la violencia familiar, las violaciones, así como los feminicidios. Todo este panorama, como hemos visto exige de la Criminología independientemente su vertiente teórica, una respuesta, donde con su entendimiento de su multidisciplinariedad, es capaz de configurar planes, programas y proyectos, ya lejos de las posiciones pasadas en las que no se tenía una metodología clara en su estructura, hoy la tenemos, por lo que nos responsabiliza como agentes de cambio ante las viejas estructuras. Con lo anterior, permite que desde la Criminología dar su posicionamiento desde análisis riguroso de la política pública, la cual despliega el gobierno mexicano en materia de la Agenda 2030, del Desarrollo Sostenible, en lo conducente al Objetivo 16, donde al momento encontramos que el proyecto con el que cuenta no es claro y con muchas dificultades para una futura medición al presentar indicadores, que no se muestra surgimiento de alguna herramienta para ello, como es la teoría de cambio o el marco lógico.

Ante este momento la academia tiene la responsabilidad de trabajar en conjunto para la transformación del conocimiento, que permita dar respuestas a la sociedad en materia de seguridad que le permita ejercer una cultura de paz libre de violencia.

\section{TRABAJOS CITADOS}

Causa Común (2020). Recuperado de: http://causaencomun. org.mx/beta/wp-content/uploads/2020/07/170720Ana\%CC\%81lisis-del-Programa-Sectorial-de-Seguridad-yProteccio\%CC\%81n-Ciudadana-2020-2024-9.56.55-1.pdf. El 12 de enero del 2021.

Constitución Política de los Estados Unidos Mexicanos.http:// www.diputados.gob.mx/LeyesBiblio/pdf_mov/Constitucion_ Politica.pdfRecuperado el 18 de mayo del 2021.

Cunjama, L., García, H. (2015). Prevención Social de las Violencias y el Delito, Análisis de los modelos teóricos. México: Instituto Nacional de Ciencias Penales.

Clarke, R., Eck, J. (2008). 60 Pasos para ser un analista delictivo. México: Instituto Nacional de Ciencias Penales.

Dammert, L. (2005). Prevención comunitaria del delito en América latina: desafíos y oportunidades. Desafíos, Vol. 13, julio-diciembre. Recuperado de: http://www.redalyc.org/ pdf/3596/359633158009.pdf . el 28 de octubre del 2020.

Estrategia Nacional para la Implementación de la Agenda 
2030 en México. Recuperado de: https://www.gob.mx/cms/ uploads/attachment/file/514075/EN-A2030Mx_VF.pdf. El 20 de enero del 2021.

García-Pablos D. (2006). Criminología. Una introducción a sus fundamentos teóricos. Perú: Ediciones luris Consulti S.A.C.

González, A., Sánchez, A. (2010). Criminología. México, D.F.: Editorial Porrúa.

Hassemer, W., Muñoz, F. (2012). Introducción a la Criminología y a la Política Criminal. Valencia, España: Tirant Lo Blanch.

Herrero, Z. (2016). Teorías Integradoras. Recuperado de: https://crimipedia.umh.es/topics/teorias-integradoras/ el 30 de mayo del 2021.

La Parra, Tortosa, J. (2003). Violencia estructural: una ilustración del concepto. Grupo de estudios de paz y desarrollo. Universidad de Alicante. Recuperado de: http://www.ugr. es/ fentrena/Violen.pdf . el 12 de julio del 2020.

Ley General para La Prevención Social de la Violencia y la Delincuencia. Recuperado de: http://www.diputados.gob. mx/LeyesBiblio/pdf/LGPSVD.pdf el 10 de marzo del 2021.

Marín Ardila, Luis Fernando. La noción de paradigma. Signo y Pensamiento, vol. XXVI, núm. 50, enero-junio, 2007 https://www.redalyc.org/pdf/860/86005004.pdf. Recuperado el 18 de mayo del 2021.

Marx, K. (2009). El Capital Tomo 1. 2ª ed. México: Fondo de Cultura Económica.

Medina, A. (2010). Políticas de seguridad ciudadana en el contexto urbano y prevención comunitaria, la experiencia anglosajona. Revista Electrónica de Ciencia Penal y Criminología. Recuperado de: http://criminet.ugr.es/ recpc/12/recpc12-02.pdf el 12 de julio del 2016.

México Evalúa (2015). Bases para la Construcción de un Índice de Cohesión Social Vecinal, Recuperado de https://www.mexicoevalua.org/mexicoevalua/wp-content/ uploads/2015/09/indice-de-cohesion-social-vecinal.pdf el 1 de enero del 2021.

Naciones Unidas (2010) https://www.unodc.org/documents/crimecongress/12th-Crime Congress/Documents/A_CONF.213_6/ V1050759s.pdf

Naciones Unidas México. Objetivos del Milenio. Recuperado de: https://www.onu.org.mx/agenda-2030/objetivos-dedesarrollo-del-milenio/ el 18 de marzo del 2021.

Observatorio de Seguridad y Justicia. Recuperado de: https:// observatorio.conl.mx/\#documents_anchor. Consultado el 8 de mayo del 2021.

Orellana W. (2012). Criminología Moderna y Contemporánea. México: Editorial Porrúa.

Organización Panamericana de la Salud. (1993). Recuperado en https://iris.paho.org/bitstream/handle/10665.2/7012/15685. pdf?sequence=1\&isAllowed=y. el 20 de mayo del 2021.

Palacios, P. (2015). Criminología contemporánea introducción a sus fundamentos teóricos. $3^{\mathrm{a}} \mathrm{ed}$. México, D.F.: Instituto Nacional de Ciencias Penales.

Pérez P., Pérez C. (2006). Curso de Criminología. $7^{\text {a }}$ Edición, Colombia: Universidad Externado de Colombia.

Pascual, B. (2006). La evaluación de la intervención comunitaria: un marco para la reflexión. Pedagogía Social: Revista Interuniversitaria. Recuperado de: http://e-spacio.uned.es/ fez/eserv/bibliuned:revistaPS-2007-14-2090/Documento.pdf el 12 de julio del 2016.

Rau, M. (2005). Prevención del Crimen Mediante el Diseño Ambiental en Latinoamérica. Un Llamado de Acción Ambiental Comunitaria. Recuperado en: http://www.bvsde. paho.org/bvsacd/cd57/rau.pdf . el 10 de marzo del 2021.

Subsecretaría de Prevención y Participación Ciudadana (SEGOB) y del Instituto Nacional Demócrata para Asuntos Internacionales (NDI). (2018). Curso 1. Bases conceptuales sobre seguridad ciudadana y prevención social de la violencia. Recuperado de https://gimpladu1618.nld.gob.mx/ img/boletines/30/5b1599107a744_boletin_30.pdf, el 20 de junio del 2018.

USAID. (2015). Manual para la capacitación en materia de prevención social de la violencia y la delincuencia. México D.F. Recuperado de: http://www.prevenciondelaviolencia. org/ebook/loche/index.html. el 13 de noviembre del 2016.

Vidaurri A. (2018). Vademécum de Criminología. México. Editorial Tirant lo Blanch. 


\section{Ana María Esquivel Hernández}

Afiliación: Universidad Autónoma de Nuevo León, México.

Doctora en Criminología, Maestría en Criminología, Licenciada en Psicología UANL, Coordinadora de la Maestría en Criminología, Catedrático en Licenciatura y Posgrado, de la Facultad de Derecho y Criminología de la UANL, así como Par Académico de Ciencias Sociales y Administrativas de los Comités Interinstitucionales para la Evaluación de la Educación Superior (CIEES) y Presidenta del Consejo Ciudadano de Seguridad Pública del Estado de NL. Correo de contacto: anaesquivel7878@hotmail.com. 